\title{
THE SPATIAL SHAPE REPRESENTATION AND 3D MODELING OF SPECIAL-SHAPE SPRING
}

\author{
Xiangchen $\mathrm{Ku}^{1,2}$, Runxiao Wang ${ }^{1}$, Jishun $\mathrm{Li}^{2}$, Dongbo Wang ${ }^{1}$ \\ ${ }^{\prime}$ School of Mechatronics Engineering,Northwestern Polytechnical University,Xi'an,China; \\ Email:kuxch@sohu.com. ${ }^{2}$ School of Mechatronics Engineering,Henan University of Science \\ and Technology,Luoyang, China.
}

Abstract: $\quad$ The solid modeling of special-shape spring is important to improve production efficiency of spring coiler. However, how to describe and represent its spatial shape is difficult because there is various special-shape spring with complex spatial shape. Consequently, the 3D modeling and visualization of specialshape spring is not easy to implement. The paper presents a solution to this problem. First, we extract four kinds of elementary shape feature and define their data structure respectively using feature technology. The special-shape spring may regarded as the combination of different elementary shape features. The dynamic linked lists are employed to record the position relation between them. Then, this article studies the 3D modeling methods of special-shape spring based on OpenGL. The emphasis is the discretization of the spring solid surface. Last, the high reality solid modeling is created with $\mathrm{C}++$ Builder.

Key words: special-shape spring; shape feature; representation; 3D modeling.

\section{INTRODUCTION}

Spring is one kind of basic mechanical parts used widely. The shapes of spring is quite different ${ }^{1}$, thus it formed various special-shape spring with complex spatial shape. Along with the development of computer technology, the solid modeling technology is playing more and more important role in the product design and manufacture process. Currently there are some papers have discussed the solid modeling for compression spring with simple shape ${ }^{2,3}$. But all these 3D modeling of spring in these papers depends on some commercial 3D modeling software. Although these software can create solid

This project is supported by the Doctoral Programme Foundation of Education Ministry of China under the grant No.20050699033.

Please use the following format when citing this chapter:

$\mathrm{Ku}$, Xiangchen, Wang, Runxiao, Li, Jishun, Wang, Dongbo, 2006, in International Federation for Information Processing (IFIP), Volume 207, Knowledge Enterprise: Intelligent Strategies In Product Design, Manufacturing, and Management, eds. K. Wang, Kovacs G., Wozny M., Fang M., (Boston: Springer), pp. 171-176. 
modeling satisfactorily, but there are still some limitations in the application of these software, such as expensive cost, inconvenient operation and high specialization. Therefore, these software is unsuitable for the design and manufacture of special-shape spring.

In fact, it is a difficult task to express the spatial shape information of special-shape spring with uniform format especially for it's complex coil end. Consequently, there is no related papers which have discussed the solid modeling of special-shape spring at present. So the effective representation of special-shape is indispensable to its solid modeling. In this paper, a solution to the solid modeling of special-shape spring is proposed and implemented by feature-based technology. Firstly, four kinds of elementary shape feature are extracted in terms of spring shape feature. The spatial shape of the special-shape spring is described with these elementary shape features. Then using OpenGL and $\mathrm{C}++$ Builder, the solid modeling for special-shape spring is researched.

\section{REPRESENTATION OF SPATIAL SHAPE OF SPECIAL-SHAPE SPRING}

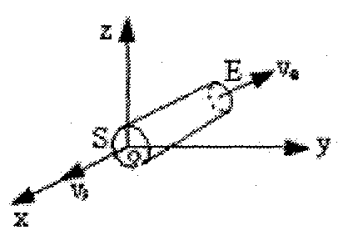

(a)column

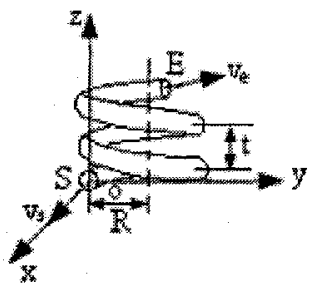

(c)helix

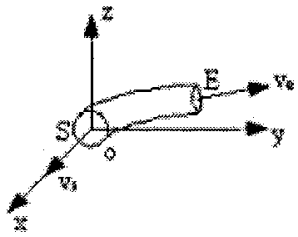

(b)torus

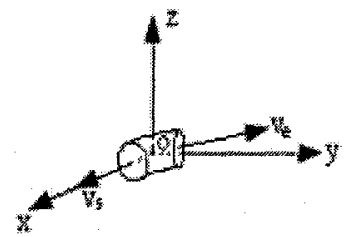

(d)bender

Figure 1.Definition of elementary shape feature

The shape feature is a basic unit to form mechanism parts. Therefore, the special-shape spring may regarded as the combination of different shape feature ${ }^{4}$. There are four kinds of elementary shapes abstracted from the special-shape spring, including helix, torus, bender and column. Figure 1 shows the definition and expression of these four elementary shape features. 

Spring

The elementary shape feature is not only the abstraction of the shape but also the representation of the spring coiling, because each of these features correspond one coiling method. Each of these elementary shape features is defined by two vertexes and direction vector. The vertex is the connection points between two different elementary shape feature. The end vertex of the previous elementary shape feature is the start vertex of next one. By using this kind of connection relations, different elementary shape features can be assembled one by one and whole spring spatial shape information can be generated finally.

In order to describe and manage the spring shape information, the data structure of elementary shape feature is defined as follows:

(1) struct FeatureBase //data structure of elementary shape feature \{

enum \{line,circle,spring,bender type; //type of elementary shape feature VERTEX *start_point; //start vertex

VECTOR *start_vector; // direction vector of start vertex

VERTEX *end_point; // end vertex

VECTOR *end_vector; //direction vector of end vertex

VERTEX * start_position;//global coordinates of start vertex

VERTEX *end_position; //global coordinates of end vertex

HELIX *helixbody; // shape parameter of helix

float angle_plane;//the angle between the two planes which the

//elementary shape feature is located

struct FeatureBase *next; //pointer of next elementary shape feature

; ;

(2)struct VERTEX //data structre of vertex position

\{

float $\mathrm{x}, \mathrm{y}, \mathrm{z} ; \quad / /$ coordinates of vertex

\};

(3)struct VECTOR //data structure of vector

\{

float nx,ny,nz; //coordinates of vector

\};

(4) struct HELIX //data structre of helix

\{

char direction; //direction of rotation

float num; $\quad / /$ number of coil

float bot_radius; //bottom radius of helix

float top_radius; //top radius of helix

float start_pitch; //start pitch of helix

float end_pitch; //end pitch of helix 
\}

\section{THE 3D MODELING OF SPECIAL-SHAPE SPRING}

OpenGL used simple geometry unit to generate complex solid. Therefore, it is need to disperse the spring solid surface into a series of slices colored by OpenGL in the modeling of spring. One spring coil can be divided into small segment of $n$, then every small segment is dispersed as equal points. The triangle planes which OpenGL can filled and colored are constructed using these equality points. Many these triangle planes are put together according to the sequence and high reality solid modeling can be obtained finally.

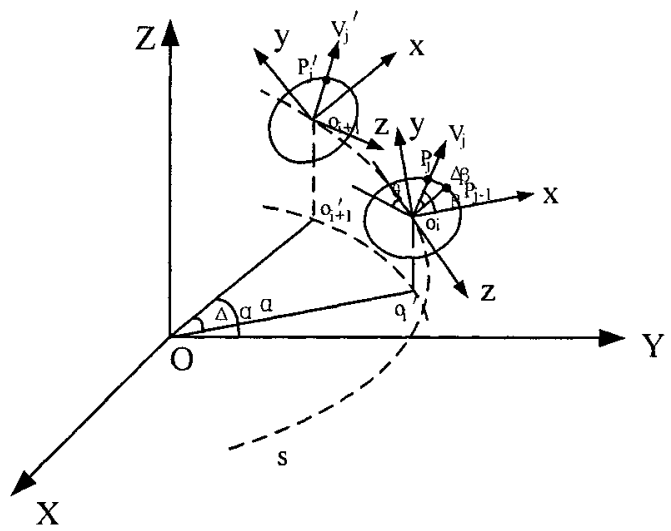

Figure 2. Discretization of spring solid surface

As shown in Fig. 2, in a global coordinate system $O X Y Z, s$ is a part of $k$ th coil centric line of a right screw spring which spiral angle is $\theta . o_{i}$ and $o_{i+1}$ is section center respectively, and their projection in the $X O Y$ plane is $o_{i}^{\prime}$ and $o_{i+1}{ }^{\prime}$. The part between $o_{i}$ and $o_{i+1}$ called $i$ th segment when one coil is divided with step length of $\Delta \alpha$. Taking $o_{i}$ as origin and establishing local coordinate system, $x$ axis is parallel to $O o_{i}^{\prime}$ and $z$ axis is paraller to normal vector of section. The $o_{i}$ global coordinate is: $x_{i}=R \cos \alpha, y_{i}=R \sin \alpha, z_{i}=(i / n+k) h$ (where, $R$ is radius of spring, $h$ is pitch, $\left.\alpha=i^{*} \Delta \alpha, i=0,1,2, \ldots\right)$. 

Spring

In xoy plane, the circumference of section circle is divided into a series of points with step length $\Delta \beta$ started from point $(d / 2,0)$ (where, $d$ is diameter of wire). The relationship between the global coordinates $(X, Y, Z)$ and local coordinates $(x, y, z)$ of the points in the circumference is as follows:

$$
\left(\begin{array}{l}
X \\
Y \\
Z
\end{array}\right)=\left(\begin{array}{ccc}
\cos X x & \cos X y & \cos X z \\
\cos Y x & \cos Y y & \cos Y z \\
\cos Z x & \cos Z y & \cos Z z
\end{array}\right)\left(\begin{array}{l}
x \\
y \\
z
\end{array}\right)+\left(\begin{array}{l}
x_{0} \\
y_{0} \\
z_{0}
\end{array}\right)
$$

In the Eq.(1), $\left(x_{0}, y_{0}, z_{0}\right)$ is the global coordinates of the origin, and the coefficient matrix is composed by direction cosine between the two coordinate system, the rest may be deduced by analogy.

As shown in Fig. 2, supposing $P_{j}$ is $j$ th point in the circumference, it's local coordinates is $((d / 2) \cos \beta,(d / 2) \sin \beta, 0)$ (where, $\left.\beta=j^{*} \Delta \beta\right)$. Using the Eq. (1), the global coordinates of this point $\left(P_{j x}, P_{j y}, P_{j z}\right)$ may be obtain as:

$$
\begin{aligned}
& P_{j x}=-(R+(d / 2) \cos \beta) \sin \alpha+(d / 2) \sin \beta \sin \theta \cos \alpha \\
& P_{j y}=(R+(d / 2) \cos \beta) \cos \alpha+(d / 2) \sin \beta \sin \theta \sin \alpha \\
& P_{j z}=(d / 2) \sin \beta \cos \theta+(i / n+k) h
\end{aligned}
$$

In order to obtain high sense of reality, it is necessary to calculate the normal vector of the disperse points. Because these points are located in the circumference of section circle, therefore the normal vector of the point equates the vector from the centre of a circle to this point. If the local unit normal vector of point $P_{j}$ is $(\cos \beta, \sin \beta, 0)$, then the global unit normal vector $V_{j}\left(V_{j x}, V_{j y}, V_{j z}\right)$ is:

$$
\begin{aligned}
& V_{j x}=\sin \beta \cos \alpha \sin \theta-\cos \beta \sin \alpha \\
& V_{j y}=\cos \beta \cos \alpha+\sin \beta \sin \alpha \sin \theta \\
& V_{j z}=\sin \beta \cos \theta
\end{aligned}
$$

Using the similar method, point $P_{j}^{\prime}$ and vector $V_{j}^{\prime}$ in another section circumference which correspond to the point $P_{j}$ and vector $V_{j}$ can be calculated. When these corresponding points is obtained, the helix curved surfaces are created using the continual small pieces of triangle surface, then the high reality solid modeling can generated by adding the illumination and material quality. The column and torus which compose special-shape spring also can be divided into small pieces of triangle surface. As a result, the solid modeling of whole spring is created. 


\section{IMPLEMENTATION AND EXAMPLES}

After obtaining the shape parameter, the paper creates the solid modeling of special-shape spring using OpenGL under the $\mathrm{C}++$ Builder 6.0 environment. Fig. 3 shows a solid modeling of spring.

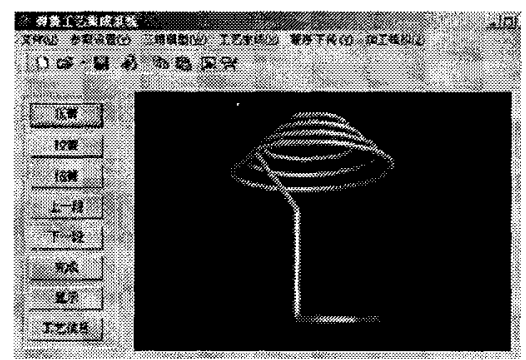

Figure 3. Solid modeling of special-shape spring

\section{CONCLUSIONS}

The advanced manufacture technology not only requires the blueprint, but also needs 3D model to describe products. This paper studies spatial shape representation and solid modeling of special-shape spring. The characteristics and contributions of the research includes (1) extracting four kinds of spring shape features and defining their data structure, (2) providing the complete shape information of spring with dynamic linked list which records the assemble process of different elementary shape features, (3)creating the solid modeling of spring, and (4)the system provides a sound foundation for the visual design and $\mathrm{CNC}$ coiling of special-shape spring.

\section{REFERENCES}

1. Michael Johnston,(2001),An engineer's guide to spring design, Wire industry, vol.68, pp.35-38.

2. Giuseppe Boschiero, (1999), Reducing setup times for critical compression springs, Wire industry, vol.66, pp 673-676.

3. YU Zhi-kun, WU Yan-ping, (2003), Study of Modeling Methods on Three Dimensional Spring Model, Journal of Kunming University of Science and Technology, vol.28, pp.4648.

4. Ting-Kuo Peng, AMY J.C.Trappey, (1996), CAD-integrated engineering-datamanagement system for spring design, Robotics and computer-integrated manufacturing, vol.12, pp.271-281. 